\title{
Efectos de la descarga en la calidad del agua a lo largo de un río mediterráneo: el río Guadaira (Sevilla)
}

\author{
Alfonso Gallardo-Mayenco*, Sonia Macías y Julia Toja \\ Departamento de Biología Vegetal y Ecología, Universidad de Sevilla, Aptdo. 1095, 41080 Sevilla, España. \\ * Autor a quién la correspondencia debe ser dirigida (algama@correo.cop.es)
}

\section{RESUMEN}

El Guadaira es un río sometido a grandes fluctuaciones de caudal a lo largo del año, con un largo periodo de estiaje. El objetivo de este trabajo era estimar la calidad de las aguas del río a partir de sus comunidades de macroinvertebrados en distintos periodos de descarga a lo largo del eje longitudinal. Para ello se estudió el número de familias, número de familias de EPT, abundancia relativa, porcentaje de taxa dominantes, y los índices IBMWP y ASPT'.

Según el IBMWP el río se encontró en la mayor parte del estudio en unas condiciones de calidad del agua buena en el tramo alto y de dudosa a muy crítica en el tramo medio-bajo. El análisis de agrupamiento de los sitios mostró que el eje del río no seguía la sucesión esperada según el gradiente de altitud o eje altitud-contaminación orgánica descrito a partir de las variables físico-químicas. Anteriores al último sitio muestreado existen numerosos molinos abandonados que provocan rápidos y torbellinos que favorecen la autodepuración del río.

La alcalinidad resultó indicadora de contaminación orgánica. Entre las familias dominantes y las variables físico-químicas se encontraron numerosas correlaciones significativas, principalmente con aquellas relacionadas con la contaminación orgánica. Caenidae y Nemouridae fueron las más correlacionadas. El análisis de agrupamiento de las familias separó en primer lugar a Oligochaeta y Chironomidae, dominantes en los sitios con mayor contenido en materia orgánica. Erpobdellidae, Physidae (Physella acuta) y Thiaridae (Melanopsis sevillensis) también estuvieron correlacionadas positivamente con la contaminación orgánica.

Las familias con mayor presencia pertenecían a dípteros y coleópteros, mostrándose como las mejor adaptadas al estrés existente en el río. La escasez de familias con alta puntuación en el IBMWP implicó que el ASPT’ no fuera dependiente de la estacionalidad.

Palabras clave: macroinvertebrados, crecidas, estiaje, IBMWP, ASPT', protocolos de calidad del agua

\begin{abstract}
The Guadaira river is subject to large fluctuations of flow through the year, with a long time of drought. The goal of this work was evaluate the water quality of the river from the macroinvertebrate communities at different times of discharge along the longitudinal course of the river. To evaluate the water quality were measured number of families, number of families of Ephemeroptera, Plecoptera and Trichoptera (EPT), relative abundance, dominant families percentages, and the IBMWP and ASPT' biological index.

In general, the river was found at conditions of good water quality at headwater and from doubtful to very critical downwater, according the IBMWP index. The cluster analysis of the sites sampled shown that the river did not follow the expected succession according to the altitudinal gradient or altitude-organic pollution axis described from physic-chemical variables. Before the last site sampled there are numerouses water mills abandoned promoting the fast circulation of the water and whirles which favoured the river self-purification.

Physic-chemical variable more correlated with the parameters of water quality was alkalinity, being an indicator of severe organic pollution. Numerouses significant correlations among the dominant families and physic-chemical variables measured, mainly with those related with organic pollution were found. Families with higher number of significant correlations were Caenidae and Nemouridae. The cluster analysis of families separed former to Oligochaeta and Chironomidae, dominant at sites with higher content of organic matter. Erpobdellidae, Physidae (Physella acuta) and Thiaridae (Melanopsis sevillensis) were too positively correlated at significant level with organic pollution.

Families with highest presence were members of Diptera and Coleoptera, seemingly the best adapted to the stress conditions existing at the Guadaira River. The lack of families with high scoring for the IBMWP index involved that ASPT' index was not seasonally dependent.
\end{abstract}

Keywords: macroinvertebrates, flood, drought, IBMWP and ASPT' index, rapid assessment protocols 


\section{INTRODUCCIÓN}

El río Guadaira constituye un paradigma de lo que es un río mediterráneo, sometido a grandes fluctuaciones de caudal a lo largo del año, con un periodo de estiaje que se prolonga de mayo a octubre. Sus rasgos ecológicos característicos son muy próximos a los descritos por Williams \& Hynes (1976) para las aguas temporales, donde el régimen natural de perturbaciones predecibles (estiajes) o más impredecibles (crecidas) es el que garantiza el mantenimiento de la biodiversidad e integridad biótica del río (Resh et al., 1988; Wootton et al., 1996). Para que una especie pueda establecerse en un cauce sometido a estas perturbaciones tendrá que presentar algunas adaptaciones como el "timing" del ciclo de vida, evitación de los periodos de flujo bajo, huevos resistentes a la desecación y capacidad de supervivencia en el hiporrheos o migración (Delucchi, 1988). Por otra parte, la inestabilidad del sustrato y limpieza del lecho por abrasión, están asociadas a las crecidas y pueden dañar a la comunidad béntica, especialmente si el sustrato es muy móvil o la intensidad de la crecida lo suficientemente alta (Rempel et al., 1999), produciendo cambios en la composición de la comunidad en los ríos mediterráneos (Gasith \& Resh, 1999).

Además, lleva unas aguas muy salinas en su cabecera debido a los terrenos yesíferos que forman su lecho. Esta salinidad va disminuyendo aguas abajo, contribuyendo a su dilución las aguas residuales procedentes de los núcleos urbanos que, a partir de Morón de la Frontera no

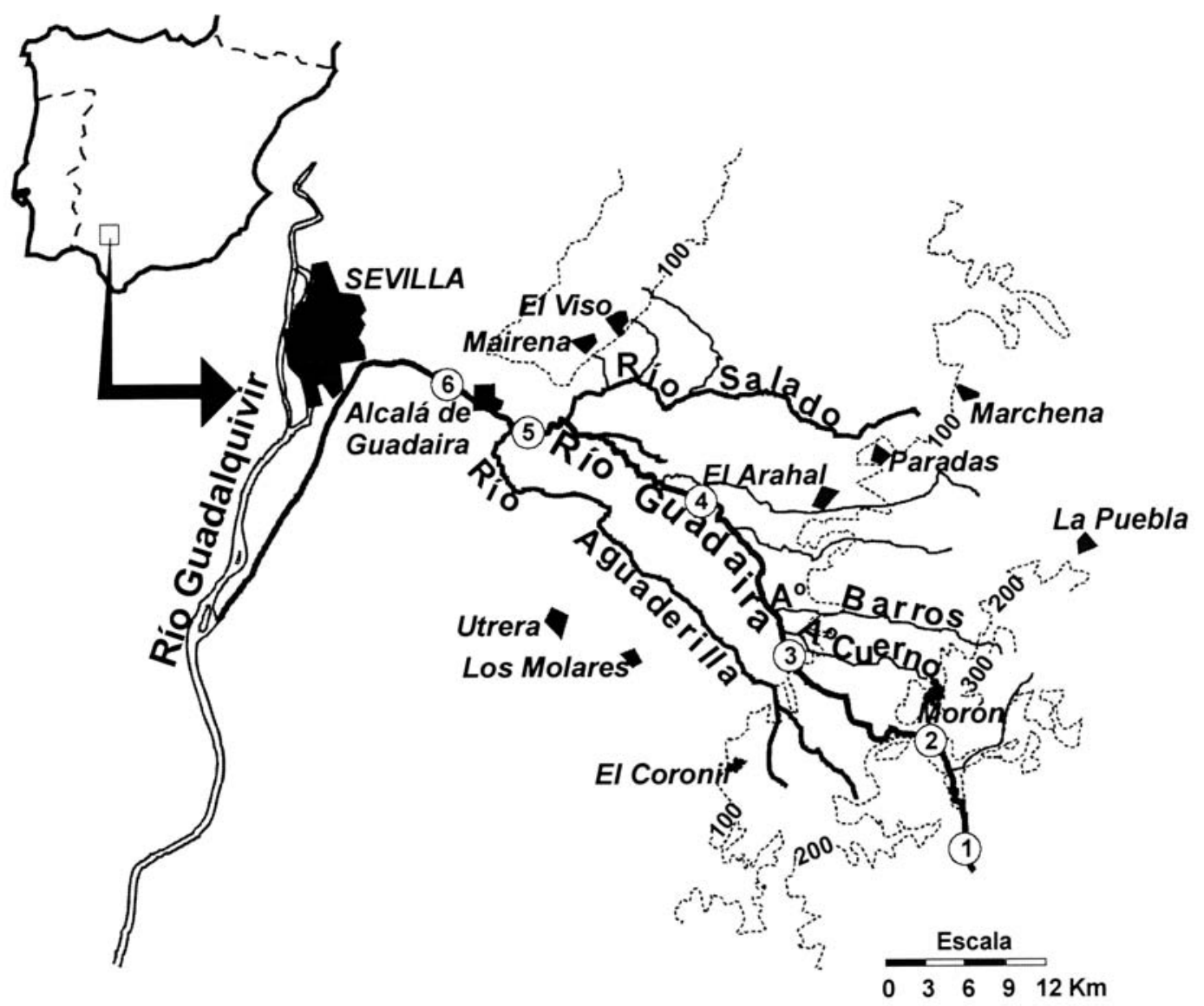

Figura 1. Cuenca del río Guadaira. Localización de las estaciones de muestreo. Guadaira River basin with the situation of sites sampled. 
dejan de fluir al cauce principal. Como consecuencia de todo ello, las especies que habitan sus aguas deben estar adaptadas a las fluctuaciones de caudal y ser capaces de tolerar los altos niveles de salinidad las que se establecen en sitios de la cabecera, donde la contaminación orgánica no es importante, o tolerar la contaminación orgánica donde la salinidad es más baja, como ocurre aguas abajo. Por ello, son muchos los grupos de macroinvertebrados acuáticos que ven restringidas sus opciones a ocupar la cuenca, caso de efemerópteros, plecópteros y tricópteros (Gallardo-Mayenco, 1991, 1993, 1994).

En este trabajo se pretende estimar la calidad de las aguas del río a partir de sus comunidades de macroinvertebrados en distintos periodos de descarga tanto en cada sitio como a lo largo del eje longitudinal, coincidiendo con unas fechas $(1996,1997)$ en que hubo una fuerte contestación social promovida por los altos niveles de contaminación del río, especialmente en la localidad de Alcalá, próxima a Sevilla, que en 2004 aún continua.

\section{ÁREA DE ESTUdIO}

El río Guadaira (Fig. 1) se caracteriza por la salinidad natural de sus aguas en la cabecera y por la irregularidad de las precipitaciones, propia de su clima mediterráneo, que pueden provocar grandes inundaciones en los periodos de lluvia, con caudales de hasta $2000 \mathrm{~m}^{3} / \mathrm{s}$, y un extenso periodo de estiaje en el que gran parte de la cuenca permanece seca, circulando por el cauce principal prácticamente nada más que aguas residuales. Por lo general, entre los meses de noviembre y enero se dan los picos de lluvia con otro más pequeño durante la primavera, mientras que en verano la lluvia es testimonial (Fig. 2). Los años en los que se realizó el muestreo fueron más lluviosos (1038 y 653,5 mm para 1996 y 1997 respectivamente) que la precipitación media mensual del periodo 1960-1990 (590.9 mm). Antes de octubre de 1996, la cuenca había tenido una primavera más lluviosa que la media (175 mm en los meses de abril y mayo frente a los $98.1 \mathrm{~mm}$

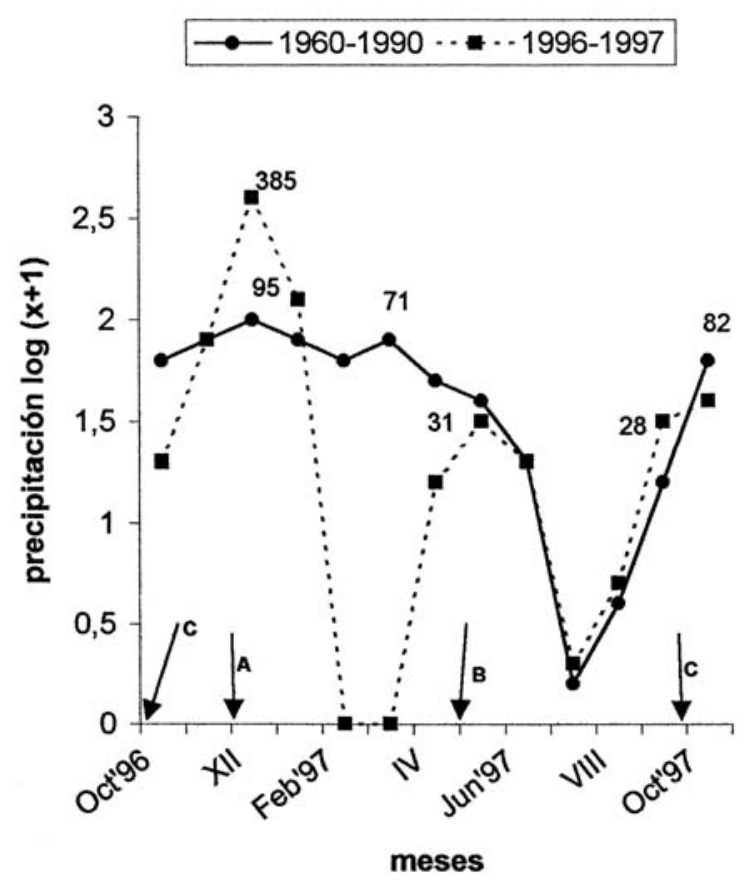

Figura 2. Precipitación media mensual $(\mathrm{mm})$ del periodo 1960-1990 y precipitación total mensual $(\mathrm{mm})$ del periodo octubre 1996-octubre 1997. Datos de la Base Aérea de Morón (Sevilla, próxima a E2). (A) crecida, (B) inicio del estiaje, (C) estiaje acusado. Se indican los valores de precipitación (mm) de cada pico. Rainfall $(\mathrm{mm})$ in the Guadaira headwater (near E2). Monthly average between 1960-1990, and total monthly between october 1996-october 1997. (A) flood, (B) drought start, $(C)$ drought pronounced. Rainfall peak $(\mathrm{mm})$ are showed for each period.

de la media para los mismos meses) pero el verano fue extremadamente seco. El invierno fue muy lluvioso, muy por encima de la media (Fig. 2), de manera que cuando se hizo el muestreo de febrero de 1997 el río en todo su eje mostraba los efectos de la inundación. La primavera fue muy seca, destacando la ausencia total de precipitación en febrero y marzo, por lo que en el muestreo de junio'97, el río ya acusaba los efectos del estiaje. En el periodo octubre 96-septiembre 97 se recogieron $713.1 \mathrm{~mm}$ de precipitación, superior a la media, de los cuales $595.3 \mathrm{~mm}(83.5 \%)$ se recogieron entre noviembre y enero.

A causa de la salinidad el río no está regulado para su uso en abastecimiento ni regadíos, por lo que la cuenca se ha convertido en un colector de los vertidos urbanos e industriales. La activi- 
dad industrial de la cuenca se reduce al tramo localizado entre Alcalá de Guadaira y Sevilla, y a las actividades de maceración de la aceituna, con la consiguiente producción de alpechines. Todos los cauces mostraron una contaminación orgánica evidente, exceptuando puntos localizados de la cabecera, aunque es en la subcuenca del Salado y en los tramos cercanos a Alcalá donde se encuentran las peores condiciones de insalubridad (Gallardo-Mayenco, 1991; Prenda \& Gallardo-Mayenco, 1996).

\section{MATERIAL Y MÉTODOS}

El río Guadaira se muestreó a lo largo de su eje longitudinal, en 6 estaciones (E1 a E6) en la dirección cabecera-salida de la localidad de Alcalá de Guadaira, en las proximidades de Sevilla (Fig. 1). E1 y E2 son las únicas estaciones que se encuentran libres de la influencia de núcleos urbanos. En E3 el río ya ha recibido parte de las aguas residuales de Morón y en E4 los aportes del Arroyo del Cuerno, receptor de otra parte de las aguas residuales de Morón, y del Arroyo de Barros, el cauce más salino de la cuenca; en E5 los aportes de la subcuenca del Arroyo Salado y en E6 ya han llegado todas las aguas de la cuenca, incluidas las aguas residuales de Alcalá, aunque aquí la presencia de algunos antiguos molinos de grano ayuda en la autodepuración del río. El uso del suelo en todas las estaciones es agrícola intensivo, excepto en E6 que es recreativo.

Las estaciones se visitaron en octubre de 1996 y febrero, junio y octubre de 1997, coincidiendo con distintas fases de descarga: estiaje acusado- crecida- inicios de estiaje- estiaje acusado. E1 no se muestreó en octubre'96 porque su incorporación al estudio se planteó con posterioridad a este muestreo y E4 y E5 no se muestrearon en octubre' 96 y octubre' 97 por la imposibilidad de acceder al tramo debido a la cantidad de cieno acumulado en sus orillas.

En cada estación, entre otras variables, se midió la velocidad de la corriente (con objeto flotante y metro rígido) y conductividad (con conductivímetro CON 611) y se tomaron muestras de agua para el posterior análisis de sólidos en suspensión, materia orgánica en suspensión, porcentaje de materia orgánica en suspensión, cloruros, alcalinidad, demanda química del oxígeno (DQO), fosfatos, nitratos, nitritos, amonios y concentración de clorofila $a$. Los métodos analíticos utilizados son los descritos en APHA (1985) y Rodier (1981).

Para el muestreo de los macroinvertebrados se utilizó una red de mano triangular de $35 \mathrm{~cm}$ de lado con luz de malla de $0.1 \mathrm{~mm}$ aproximadamente y mango de $1 \mathrm{~m}$. En cada estación se tomaron muestras de todos los mesohábitats encontrados en base a diferencias en el sustrato dominante, velocidad de la corriente y presencia/ausencia de vegetación, a lo largo de unos $30 \mathrm{~m}$ del tramo estudiado. En cada uno de estos mesohabitats se hicieron de 2 a 5 mangadas dependiendo del número de ejemplares distintos observados a simple vista, siendo cada mangada de una duración efectiva de $30 \mathrm{~s}$. Las abundancias relativas, expresada en individuos por unidad de esfuerzo (i.p.u.e.), se obtenían dividiendo el número de ejemplares recogidos por el número de mangadas realizadas, de manera que fueran comparables entre los sitios y fechas. La utilidad de estos muestreos cualitativos para esta clase de trabajos ha sido constatada por Storey et al. (1991), Lenat (1984) y Rodriguez \& Wright (1991).

La identificación taxonómica se hizo a nivel de familia, suficiente para conocer la biodiversidad de la comunidad y la calidad del agua (Bournaud et al., 1996; Rieradevall et al., 1999).

A partir de las muestras de macroinvertebrados se hizo la valoración biológica de la calidad de las aguas según los parámetros métricos descriptivos de la comunidad: número de familias, número de familias de efemerópteros, plecópteros y tricópteros (EPT), abundancia relativa y porcentaje de taxa dominantes (Resh \& McElravy, 1993; Resh, 1994). También se calcularon los índices IBMWP y ASPT' de AlbaTercedor \& Sánchez-Ortega (1988). En el cálculo del IBMWP se utilizó la versión de Alba-Tercedor (1996) con algunas modificaciones: la puntuación de Astacidae pasó de 8 a 4, 
ya que la especie presente en el río Guadaira era Procambarus clarkii y se incluyó a Muscidae con valor 2 y Noteridae con valor 5. Los Oligoquetos se consideraron como una familia.

Para conocer que variables ambientales de las consideradas eran más representativas en el periodo de estudio, se hizo un análisis de componentes principales (ACP) sobre la matriz de la media de los datos físico-químicos. En cada muestreo se calcularon las correlaciones entre las variables físico-químicas medidas (excepto altitud), los parámetros descriptivos de las comunidades, los índices biológicos, y las familias 3TD\% (modificado del 5TD\%). Este último valor indica el porcentaje acumulado sobre la abundancia total de la muestra por las tres familias dominantes y constituye una medida adicional de la uniformidad de la comunidad (Sponseller et al., 2001). También se calcularon los coeficientes de similitud de Jaccard entre las estaciones a partir de los datos globales del estudio, y a partir de la matriz obtenida se obtuvo el agrupamiento para los sitios.

A partir de la matriz abundancias relativas de las familias $\mathrm{X}$ estaciones, se obtuvo el agrupamiento de las familias de macroinvertebrados en el global del estudio. Para ello, solo se tuvieron en consideración aquellas familias que en el total del estudio se encontraron al menos en tres estaciones o que su abundancia relativa supusiera al menos el $0.5 \%$ de la abundancia total, basándonos para ello en las abundancias tan elevadas que mostraron algunas familias.

Todos los valores fueron convertidos a la forma $\log (x+1)$ con la finalidad de homogeneizar las muestras. Para los análisis estadísticos se utilizó el programa STATISTICA (1984-85).

\section{RESULTADOS Y DISCUSIÓN}

\section{Variables físico-químicas}

Las medias de las más bajas de todas las variables medidas, excepto sólidos en suspensión y porcentaje de materia orgánica, se midieron en E1. Los valores más altos de sólidos en sus- pensión y conductividad se encontraron en E2, mientras que los más altos de las variables relacionadas con la carga orgánica se dieron a partir de E3, especialmente entre las estaciones E4 a E6, donde se midieron los valores más altos de alcalinidad, fosfato y amonio (E4 y E5), DQO (E4 y E6) y nitritos (E4, E5 y E6) (Tabla 1). Aunque toda la cuenca se ve sometida a la agricultura intensiva, con los efectos potenciales que ello implica (Barton, 1996), es a partir de E2 donde el río empieza a recibir las aguas residuales de la cuenca.

El dato más relevante de las primeras condiciones de estiaje (octubre'96) fueron las concentraciones de nitrato medidas $(1761,2836 \mathrm{y}$ $1522 \mu \mathrm{g}$-at/L en E2, E3 y E6 respectivamente) coincidentes con graves problemas de abastecimiento en algunas localidades de la cuenca y las concentraciones de clorofila $a$ medidas en E3 y E5 (102 y $42 \mathrm{mg} / \mathrm{L}$ respectivamente). También se midieron los valores más altos de conductividad del estudio: 5.2 y $3.5 \mathrm{mS} / \mathrm{cm}$ en E2 y E3 respectivamente y los de cloruros para las cuatro estaciones muestreadas en esa fecha.

En el periodo de descarga (febrero'97), se midieron las velocidades más altas para E1 y E2, y la del total del estudio en E6 $(1 \mathrm{~m} / \mathrm{s})$. Se produjo un descenso en las concentraciones de nitrato y clorofila $a$ y en la de los parámetros relacionados con la salinidad. Por el contrario, se encontraron los valores de fosfato más altos en E1 y E2 (26 y $53 \mu \mathrm{g}$-at/L respectivamente) y de sólidos en suspensión en E2 y E3 (261 y $98 \mathrm{mg} / \mathrm{L}$ respectivamente). La alcalinidad fue muy similar entre las estaciones, con rango de 5 (E1) a $6.3 \mathrm{meq} / \mathrm{L}(\mathrm{E} 5)$.

En el inicio del estiaje (junio'97) se produjo un ligero aumento de la conductividad en E2 y un descenso de los sólidos en suspensión en E2 y E3 frente a un aumento en el resto de las estaciones (los máximos encontrados en cada una de ellas). Los valores de nitrato siguieron descendiendo, mientras que los de amonio empezaron a ser importantes, encontrando el valor máximo de E1 (69 $\mu \mathrm{g}$-at/L). En E3 y E6 se midieron las velocidades más altas $(0.7 \mathrm{~m} / \mathrm{s})$, mientras que en E1 fue nula. 
En el último muestreo, estiaje acusado (octubre'97), el río había recibido los vertidos de alpechines y en el curso medio-bajo del río las aguas eran de color negro, encontrándose en E5 desprendimiento de metano. Subió el fosfato en todas las estaciones, encontrando las concentraciones más altas en E3, E4, E5 y E6 (62, 116, 108 y 110 $\mu \mathrm{g}$-at/L respectivamente), y el nitrógeno se encontró principalmente en su forma más reducida con valores excepcionalmente altos en el curso bajo (1052, 908 y $523 \mu \mathrm{g}$-at/L para E4, E5 y E6 respectivamente). La alcalinidad también fue muy alta pasando a un rango de E1 a E6 de 5.1-11 meq/L. El porcentaje de materia orgánica en suspensión llegó a ser del 82\% (E4), 77\% (E5) y 69\% (E6). Las velocidades más altas seguían siendo las medidas en E3 y E6 con 0.7 m/s.

El ACP realizado con las medias de cada variable físico-química (Fig. 3) ordenó dichas variables de manera que el eje I aparecía definido por la altitud en el extremo negativo ( $\mathrm{r}=$ $0.94, \mathrm{p} \leq 0.005$ ), situándose en el extremo opuesto del eje fosfato $(\mathrm{r}=0.96, \mathrm{p} \leq 0.005)$, nitrito

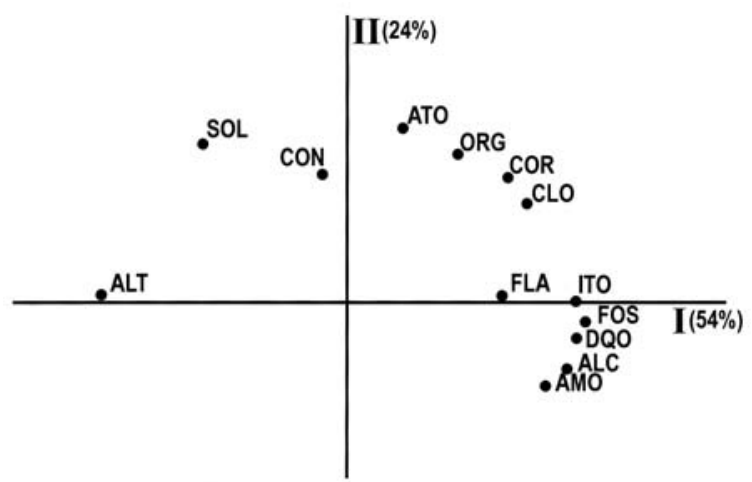

Figura 3. Representación gráfica de las correlaciones entre las medias de los parámetros físico-químicos medidos sobre los dos primeros ejes del ACP (ALT: altitud, SOL: sólidos en suspensión, COR: velocidad superficial de la corriente, CON: conductividad, CLO: cloruro, ALC: alcalinidad, DQO: demanda química del oxígeno, FOS: fosfato, ATO: nitrato, ITO: nitrito, AMO: amonio, ORG: contenido en materia orgánica, FLA: concentración clorofila $a$ ). Plot of the scores of the physic-chemical variables measured on the former two principal component axes (ALT: altitude, SOL: suspended solids, COR: flow velocity, CON: conductivity, CLO: chloride, ALC: alcalinity, DQO: permanganate value, FOS: phosphate, ATO: nitrate, ITO: nitrite, AMO: ammonium, ORG: organic matter content, FLA: chlorophyl a concentration). $(\mathrm{r}=0.94, \mathrm{p} \leq 0.005), \mathrm{DQO}(\mathrm{r}=0.92, \mathrm{p} \leq 0.01)$, alcalinidad $(\mathrm{r}=0.88, \mathrm{p} \leq 0.01)$ y amonio $(\mathrm{r}=0.81$, $\mathrm{p} \leq 0.05$ ), es decir se trata de un eje altitud-contaminación orgánica, con las estaciones sin contaminación en la cabecera del río. El eje II aparece definido por sólidos en suspensión ( $\mathrm{r}=0.80$, $\mathrm{p} \leq 0.05)$, nitratos $(\mathrm{r}=0.87, \mathrm{p} \leq 0.01)$ y contenido de materia orgánica en suspensión $(\mathrm{r}=0.73$, $\mathrm{p} \leq 0.05$ ), en el extremo positivo.

\section{Parámetros descriptivos de la calidad de las aguas}

\section{Abundancias}

Las mayores abundancias se encontraron aguas abajo, en E3, E5 y E6 (2233, 2685, 2120 i.p.u.e., respectivamente) (Tabla 2). No se encontró ninguna relación significativa entre la abundancia y las variables físico-químicas medidas lo que estaría de acuerdo con Silva \& Maltchik (2000) al sugerir que inundación y estiaje son los factores más importantes para explicar los patrones de sucesión de los macroinvertebrados y con Karr (1999) al señalar que la abundancia raramente es un indicador fidedigno de la influencia humana.

\section{Número de familias y familias EPT}

En el total del trabajo se encontraron 45 familias (considerando a Oligoquetos como una familia), de las cuales 8 estaban incluidas en el grupo EPT. Los valores más altos se encontraron en E1 y E2 (27 y 21 familias, respectivamente) y para las familias EPT (5) se dio en E1 (Tabla 2). Sin embargo, se deben considerar las limitaciones que supone la salinidad natural para muchos efemerópteros, plecópteros y tricópteros (Gallardo-Mayenco, 1994).

\section{IBMWP y ASPT'}

Los valores más altos del índice IBMWP se encontraron en E1 y E2 (104 en E1 en junio'97, Tabla 2). A partir de E3 el valor de este índice desciende, llegando a sus valores más bajos en E4 para, desde ahí, iniciar una recuperación más manifiesta en E6. Considerando a partir de 80 el valor de IBMWP indicador de buena calidad del 


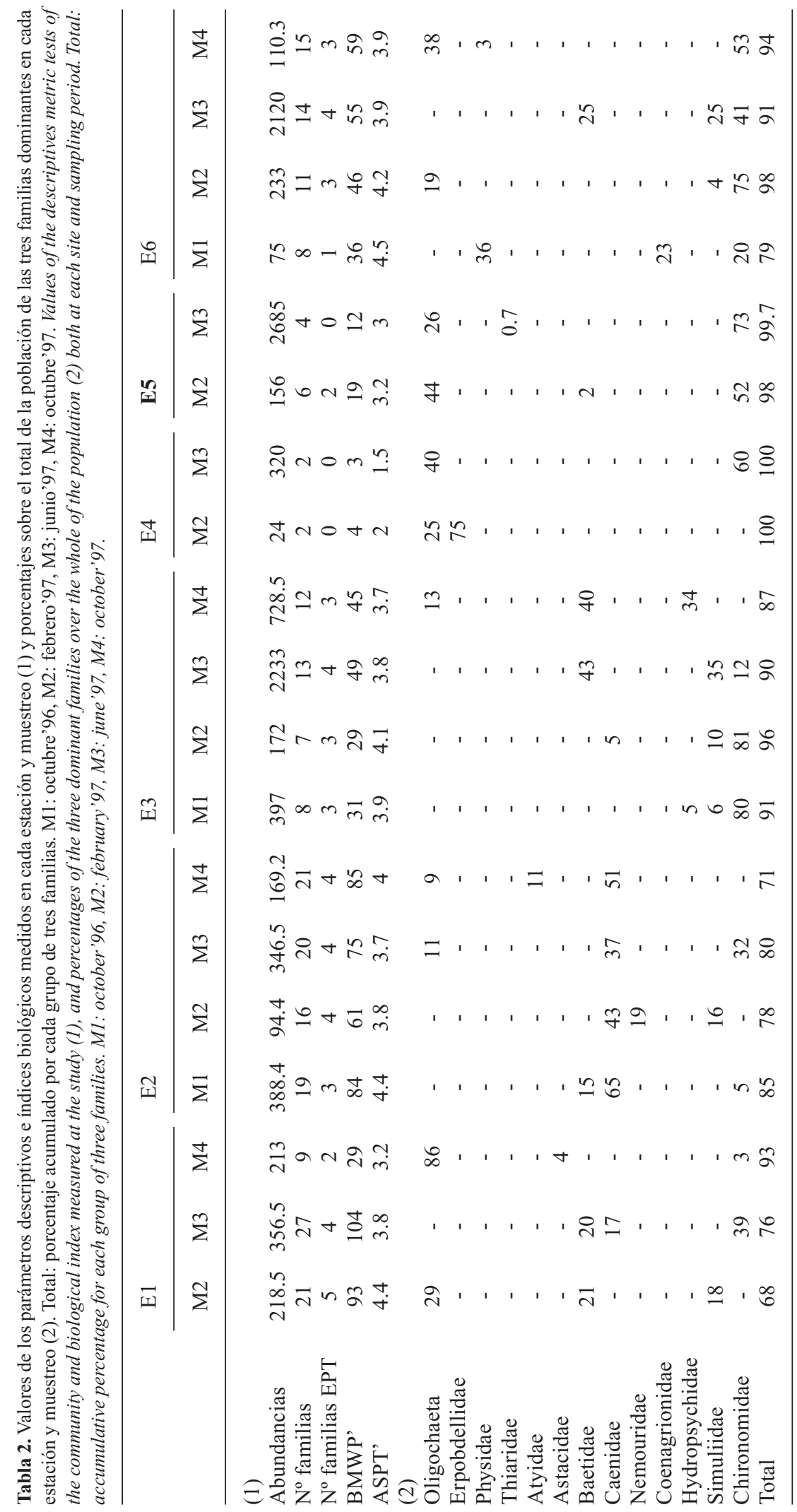




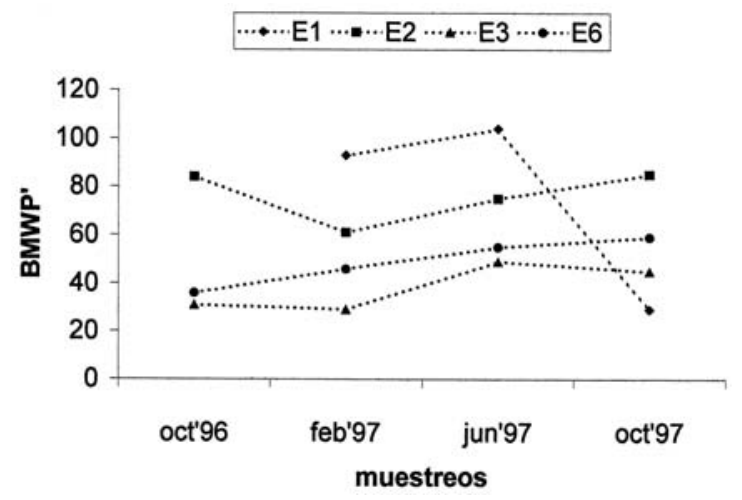

Figura 4. Valores del índice IBMWP en E1, E2, E3 y E6 en el periodo de estudio. IBMWP index values at the sites 1, 2, 3, and 6 through the sampling period.

agua en los ríos mediterráneos (Rieradevall et al., 1999), los resultados separaban dos tramos bien diferenciados: E1 y E2 con calidad del agua, en general, buena y el tramo medio-bajo con aguas contaminadas (E3 y E6) o fuertemente contaminadas (E4 y E5) (Tabla 2, Fig. 4). Por otra parte, la abrasión del lecho por las crecidas puede ser la causa de que los valores del índice IBMWP sean más altos en condiciones de estiaje que en la de descarga en la mayoría de las estaciones (Tabla 2), aunque las inundaciones tengan más incidencia sobre las abundancias que sobre las familias y la capacidad de dilución del río sea más baja durante el estiaje (Palmer et al., 1996; Gasith \& Resh, 1999).

Los valores de ASPT' fueron similares en todas las estaciones, excepto en E4 y E5 (Tabla 2), posiblemente debido a la escasez en el río Guadaira de familias con puntuación relevante en el índice IBMWP.

El estancamiento de las aguas, junto a la descomposición de la abundante materia vegetal (especialmente Nasturtium officinalis) que invadía el cauce en los meses anteriores y el aumento de la temperatura (Covich \& Crowl, 2002), pudo ser la causa de los resultados encontrados para los parámetros descriptivos de la comunidad en E1 en octubre'97 (Tabla 2).

Se encontraron algunas correlaciones significativas (todas de signo negativo) entre los parámetros descriptivos y las variables físico-quími- cas medidas. Así, número de familias lo estuvo con DQO $(\mathrm{r}=0.999, \mathrm{p} \leq 0.01)$ en octubre'96 y alcalinidad $(r=0.916, p \leq 0.05)$ en junio'97; familias EPT con alcalinidad $(r=0.901, p \leq 0.05)$ en junio'97; BMWP' con alcalinidad ( $\mathrm{r}=0.890$, $\mathrm{p} \leq$ 0.05) en junio'97; y ASPT' con nitratos $(r=0.997, p \leq 0.05)$ en octubre'96 y con cloruros $(r=0.992, p \leq 0.01)$ en octubre'97.

\section{Familias dominantes}

A lo largo del estudio se encontraron variaciones en las familias dominantes y el porcentaje de abundancia que acumulaban, aunque Oligoquetos y Chironomidae fueron dominantes en la mayoría de los casos, coincidiendo con las situaciones de alta contaminación orgánica (Tabla 2). En E1, cuando se encontró gran cantidad de materia orgánica acumulada en el lecho procedente de la vegetación en descomposición, los Oligoquetos alcanzaron un porcentaje propio de aguas muy contaminadas. En E2 Caenis luctuosa fue dominante en todo el muestreo, representando en algunos muestreos más del $50 \%$ de la comunidad. Baetidae fue dominante en algunos muestreos en E3, en competencia con Simuliidae e Hydropsychidae. Se encontraron algunas dominancias aisladas de Erpobdellidae en E4, y en octubre'96 de Physellla acuta en E6, coincidiendo con un bloom de Lemna gibba. En E4 y E5 la población de Chironomidae estaba formada casi totalmente por Chironomus gr thumni, mientras que en el resto de las estaciones dominaba Orthocladiinae.

En los cuatro muestreos se encontraron algunas correlaciones significativas entre las familias dominantes y las variable físico-químicas medidas (Tabla 3). Caenidae presentó el mayor número de correlaciones significativas, negativas con las variables asociadas a la contaminación orgánica y positivas con sólidos en suspensión y conductividad. Nemouridae y Simuliidae fueron otras familias correlacionadas negativamente con la contaminación orgánica, así como con cloruros (ambas familias fueron más abundantes en las estaciones donde se midió la menor concentración de cloruros, sobre todo Nemouridae en E1). Baetidae también estuvo correlacionada negativamente, aunque en menor 
Tabla 3. Correlaciones (R) significativas encontradas en cada muestreo entre las familias dominantes y las variables físico-químicas medidas. En paréntesis, el signo de la correlación, ${ }^{*} \mathrm{p} \leq 0.05 ;{ }^{*} \mathrm{p} \leq 0.01 ; * * \mathrm{p} \leq 0.005$. Significant correlations found among the dominant families and physico-chemical variables measured, at each sampling period. The sign of each correlation is showed in brackets, ${ }^{*} p \leq 0.05, * * p \leq 0.01, * * * p \leq$ 0.005 .

\begin{tabular}{|c|c|c|c|c|}
\hline & & $\mathbf{r}$ & variable & muestreo \\
\hline \multirow[t]{3}{*}{ Oligochaeta } & $(-)$ & $0.838^{*}$ & sólidos en suspensión & febrero'97 \\
\hline & $(+)$ & $0.859 *$ & \% materia orgánica & febrero'97 \\
\hline & $(-)$ & $0.959 *$ & sólidos en suspensión & octubre'97 \\
\hline Thiaridae & $(+)$ & $0.827^{*}$ & nitritos & junio’97 \\
\hline Physidae & $(+)$ & $0.954 *$ & amonios & octubre'97 \\
\hline Atyidae & $(+)$ & $0.980 *$ & conductividad & octubre'97 \\
\hline Astacidae & $(-)$ & $0.959 *$ & cloruros & octubre'97 \\
\hline \multirow[t]{2}{*}{ Baetidae } & $(-)$ & $0.817^{*}$ & conductividad & febrero'97 \\
\hline & $(-)$ & $0.896^{*}$ & fosfatos & febrero'97 \\
\hline \multirow[t]{7}{*}{ Caenidae } & $(+)$ & $0.930 * *$ & sólidos en suspensión & febrero'97 \\
\hline & $(-)$ & $0.951 * * *$ & \% materia orgánica & febrero'97 \\
\hline & $(-)$ & $0.973 * * *$ & fosfatos & junio'97 \\
\hline & $(-)$ & $0.870 *$ & nitratos & junio’97 \\
\hline & $(-)$ & $0.921 * *$ & clorofila $a$ & junio’97 \\
\hline & $(-)$ & $0.856^{*}$ & alcalinidad & junio’97 \\
\hline & $(+)$ & $0.997 * * *$ & conductividad & octubre'97 \\
\hline \multirow[t]{4}{*}{ Nemouridae } & $(-)$ & $0.965 * * *$ & cloruros & febrero'97 \\
\hline & $(-)$ & $0.962 * * *$ & DQO & febrero'97 \\
\hline & $(-)$ & $0.974 * * *$ & nitritos & febrero'97 \\
\hline & $(-)$ & $0.876^{*}$ & amonios & febrero'97 \\
\hline \multirow[t]{3}{*}{ Simuliidae } & $(-)$ & $0.997^{*}$ & amonios & octubre'96 \\
\hline & $(-)$ & $0.836^{*}$ & cloruros & febrero'97 \\
\hline & $(-)$ & $0.832 *$ & nitritos & febrero'97 \\
\hline \multirow[t]{2}{*}{ Chironomidae } & $(+)$ & $0.972 *$ & \% materia orgánica & octubre'97 \\
\hline & $(+)$ & $0.977^{*}$ & amonios & octubre'97 \\
\hline
\end{tabular}

medida con la contaminación orgánica y salinidad. Por el contrario, Oligoquetos, Thiaridae (Melanopsis sevillensis en E5), Physidae (Physella acuta) y Chironomidae estuvieron correlacionadas positivamente con la contaminación orgánica, mientras que Astacidae y Atyidae estuvieron más ligadas a la salinidad (Tabla 3).

El análisis de agrupamiento de las familias (Fig. 5) separó en primer lugar a Oligochaeta y Chironomidae, dominantes en los sitios con mayor contenido en materia orgánica (E4 y E5) del resto de las familias, seguidas de Hydropsychidae, Simuliidae y Baetidae, que tuvieron mayor relevancia en E3, y Caenidae, dominante en E2.

En las cuatro campañas de muestreo fueron los dípteros los mejor representados (5 familias de un total de 19, 9 de 28,13 de 37 y 7 de 29 , en orden cronológico de los muestreos). Las familias de dípteros, heterópteros y coleópteros acumulaban unos porcentajes de 59 y 52 en los muestreos de junio'97 y octubre'97 respectivamente, mientras que en febrero'97 este valor era del 39\%. Por el contrario, en febrero'97 se encontraron en todo el río cuatro familias con una puntuación importante en el cálculo del índice IBMWP (Capniidae, Nemouridae, Libellulidae y Limnephilidae), en junio'97 Perlodidae y Libellulidae y en los dos muestreos de octubre (96 y 97) solo apareció Libellulidae. El río Guadaira parece ser ocupado más exitosamente por taxones mejor adaptados a las condiciones de avenidas y estiaje, además de la contaminación orgánica y salinidad. Este estrés lleva a las comunidades a una serie 


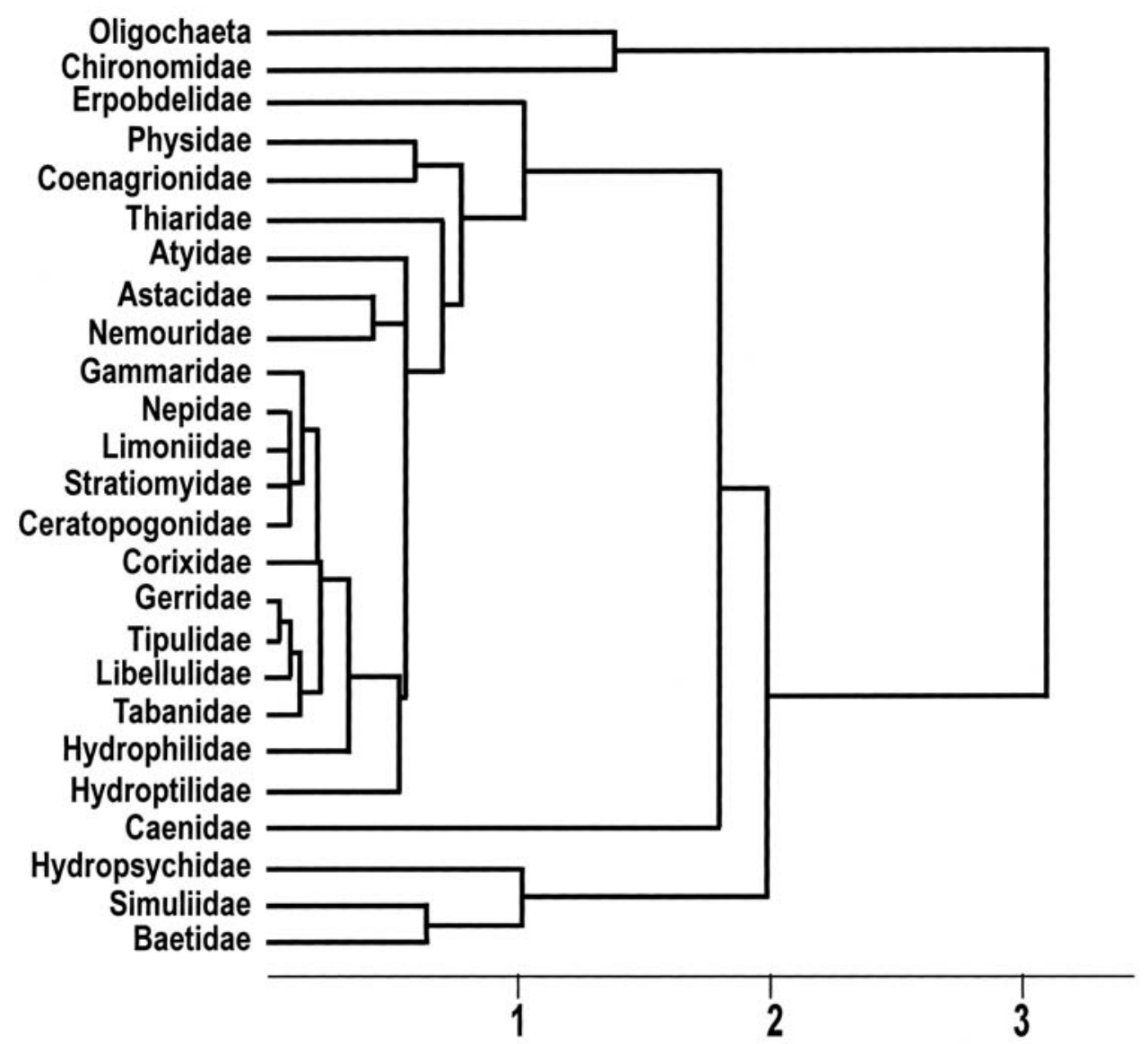

Figura 5. Clasificación de las familias basada en sus abundancias en el global del estudio. Cluster analysis of the families based in their abundances according to the whole of the study.

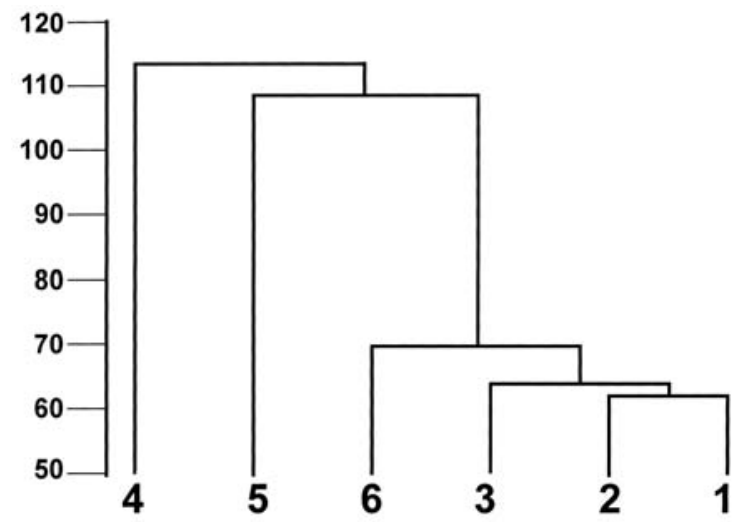

Figura 6. Clasificación de las seis estaciones de muestreo basada en la matriz de los coeficientes de similitud de Jaccard calculados a partir del total de datos. Cluster analysis of the six sites sampled, based on the matrix of the coeficient of similarity of Jaccard calculated from the whole of data. de adaptaciones de manera que durante las inundaciones persisten a través de la resilencia, mientras que en el estiaje los taxones más resistentes tendrán éxito en la colonización (Miller \& Golladay, 1996) y a la regulación de los ciclos biológicos y estrategia reproductiva a la escala temporal de variabilidad (Sabater et al., 1992; Statzner et al., 1997).

En el análisis de agrupamiento de los sitios realizado a partir de la matriz de similitud (Fig. 6) la estación E6 apareció más próxima a E1, E2 y E3, mientras que E4 y E5, más contaminadas, estaban más alejadas de ellas. La presencia de numerosos molinos abandonados antes de E6, con rápidos y torbellinos que favorecen la oxigenación del agua, explicaría la mejoría de la calidad del agua en esta estación. 


\section{CONCLUSIONES}

La alcalinidad, muy determinada por la materia orgánica (Oliver et al., 1983, tomado de Mulholland, 1997), fue la variable físico-química significativamente más correlacionada, negativamente, con los parámetros descriptores de la comunidad, mostrándose en este estudio como indicadora de contaminación orgánica.

El índice IBMWP separó dos tramos bien diferenciados en el río Guadaira: la cabecera, (E1 y E2), con una calidad del agua, en general, buena, y el tramo medio-bajo con aguas contaminadas (E3 y E6) o fuertemente contaminadas (E4 y E5) (Tabla 2, Fig. 4).

No se encontraron variaciones estacionales importantes del índice ASPT', de acuerdo con lo encontrado por otros autores (Zamora-Muñoz et al., 1995).

Baetidae, Caenidae, Nemouridae y Simuliidae estuvieron significativamente relacionadas, negativamente, con las variables físico-químicas indicadoras de la contaminación orgánica, mientras que Oligoquetos, Thiaridae, Chironomidae y Physidae lo fueron positivamente. La preponderancia de Oligoquetos y Ch. gr thumni (en E4 y E5) indicaría altos contenidos de materia orgánica en el sedimento (Angradi, 1999; Podraza, 2002). También Erpobdellidae y Physidae (Physella acuta) serían buenas indicadoras de contaminación orgánica en el área de estudio (Fruget, 1991 y Mellado et al., 2002), al igual que Melanopsis sevillensis (Thiaridae).

En general, en este trabajo coincidiendo con Karr (1999) se pone de manifiesto como el porcentaje acumulado de las tres familias dominantes está relacionado positivamente con la contaminación (Tabla 2).

El hecho de que sean coleópteros y dípteros los que aportan mayor número de familias $(6 \mathrm{y}$ 14 respectivamente) en el estudio, parece indicar que sean estos grupos los mejor adaptados a las condiciones de avenidas y estiaje, además de a la contaminación orgánica y salinidad.

Se pone en evidencia la extraordinaria capacidad de autodepuración del río Guadaira, facilitada por los numerosos molinos en desuso existen- tes en las proximidades de la localidad de Alcalá de Guadaira, por lo que a la hora de poner en práctica cualquier tipo de medidas de recuperación éstos deberían tenerse en consideración.

\section{AGRADECIMIENTOS}

Dos revisores anónimos contribuyeron con sus sugerencias y comentarios a mejorar una primera versión del manuscrito.

\section{BIBLIOGRAFÍA}

ALBA-TERCEDOR, J. 1996. Macroinvertebrados acuáticos y calidad de las aguas de los río. $I V$ SIAGA, II: 203-213.

ALBA-TERCEDOR, J. \& A. SÁNCHEZ-ORTEGA. 1988. Un método rápido y simple para evaluar la calidad biológica de las aguas corrientes basado en el de Hellawell (1978). Limnetica, 4: 51-56.

AMERICAN PUBLIC HEALTH ASSOCIATION. 1985. Standard methods for the examination of water and wastewater. APHA, AWWH, WPCF.

ANGRADI, T. R. 1999. Fine sediment and macroinvertebrate assemblages in Appalachianstreams: a field experiment with biomonitoring applications. J. N. Am. Benthol. Soc., 18: 49-66.

BARTON, D. R. 1996. The use of percent model affinity to assess the effects of agriculture on benthic invertebrate communities in headwater streams of southern Ontario, Canada. Freshwater Biology, 36: 397-410.

BOURNAUD, M., B. CELLOT, P. RICHOUX \& A. BERRAHOU. 1996. Macroinvertebrate community structure and environmental characteristics along a large river: congruity of patterns for identification to species or family. J. N. Am. Benthol. Soc., 15: 232-253.

COVICH, A. P. \& T. A. CROWL. 2002. Reorganization of benthic stream food webs in response to drought-altered population densities: effects on rainforest streams. Verh. Internat. Verein. Limnol., 28: 1172-1175.

DELUCCHI, C. M. 1988. Comparison of community structure among streams with different temporal flow regimes. Can. J. Zool., 66: 579-586.

FRUGET, J. F. 1991. The impact of river regulation on the biotic macroinvertebrate communities of 
the Lower Rhône, France. Reg. Rivers: Res. \& Manage., 6: 241-255.

GALLARDO-MAYENCO, A. 1991. Respuesta de macroinvertebrados fluviales a la salinidad. Comparación de las cuencas de los ríos Guadaira y Guadalete. Tésis Doctorales en microfichas $\mathrm{n}^{\circ}$ 33 (1993). Publicaciones de la Universidad de Sevilla.

GALLARDO-MAYENCO, A. 1993. Macroinvertebrate associations in two basins of SW Spain. Archiv Hydrobiol., 127: 473-483.

GALLARDO-MAYENCO, A. 1994. Freshwater macroinvertebrate distribution in two basins with different salinty gradients (Guadalete and Guadaira river basins, south-western Spain). Int. J. Salt Lake Res., 3: 75-91.

GASITH, A. \& V. H. RESH. 1999. Streams in Mediterranean climate regions: abiotic influences and biotic responses to predictable seasonal events. Annu. Rev. Ecol. Syst., 30: 51-81.

KARR, J. R. 1999. Defining and measuring river health. Freshwater Biology, 41: 221-234.

LENAT, D. R. 1984. Agriculture and stream water quality: a biological evaluation of erosion control practices. Environ. Management, 8: 333-344.

MELLADO, A., M. L. SUÁREZ, J. L. MORENO \& M. R. VIDAL-ABARCA. 2002. Aquatic macroinvertebrate biodiversity in the Segura River Basin (SE Spain). Verh. Internat. Verein. Limnol., 28: 1157-1162.

MILLER, A. M. \& S. W. GOLLADAY. 1996. Effects of spates and drying on macroinvertebrate assemblages of an intermittent and a perennial prairie stream. J. N. Am. Benthol. Soc., 15: 670-689.

MULHOLLAND, P. J. 1997. Dissolved organic matter concentration and flux in streams. J. N. Am. Benthol. Soc., 16: 131-141.

PALMER, M. A., P. ARENSBURGER, A. P. MARTÍN \& D. W. DENMAN. 1996. Disturbance and patch-specific responses: the interactive effects of woody debris and floods on lotic invertebrates. Oecologia, 105: 247-257.

PODRAZA, P. B. C. 2002. The intermediate-disturbance hypothesis in urban streams. Verh. Internat. Verein. Limnol., 28: 451-454.

PRENDA, J. \& A. GALLARDO-MAYENCO. 1996. Self-purification, temporal variability and the macroinvertebrate community in a small lowland Mediterraenan streams receiving crude domestic sewage effluents. Archiv Hydrobiol., 136: 159170.
REMPEL, L. L., J. S. RICHARDSON \& M. C. HEALEY. 1999. Flow refugia for benthic macroinvertebrates during flooding of a large river. J. N. Am. Benthol. Soc., 18: 34-48.

RESH, V. H. 1994. Variability, accuracy, and taxonomic costs of rapid assessment approaches in benthic macroinvertebrate biomonitoring. Boll. Zool., 61: 375-383.

RESH, V. H., A. V. BROWN, A. P. COVICH, M. E. GURTZ, H. W. LI \& G. W. MINSHALL. 1988. The role of disturbance in stream ecology. $J . N$. Am. Benthol. Soc., 7: 433-455.

RESH, V. H. \& E. P. McELRAVY. 1993. Contemporary quantitative approaches to biomonitoring using benthic macroinvertebrates. In: Freshwater Biomonitoring and Benthic Macroinvertebrates. D. M. Rosenberg \& V. H. Resh (eds.): 159-194. Chapman \& Hall.

RIERADEVALL, M., N. BONADA \& N. PRAT. 1999. Community structure and water quality in the Mediterranean streams of a natural park (St. Llorenç del Munt, NE Spain). Limnetica, 17: 4556.

RODIER, J. 1981. Análisis de las Aguas. Omega, Barcelona.

RODRIGUEZ, P. \& J. F. WRIGHT. 1991. Description and evaluation of a sampling strategy for macroinvertebrate communities in Basque rivers (Spain). Hydrobiologia, 213: 113-124.

SABATER, S., H. GUASCH, E. MARTÍ, J. ARMENGOL, M. VILA \& F. SABATER. 1992. The Ter, a mediterranean river system in Spain. Limnetica, 8: 141-149.

SILVA, M. I. \& L. MALTCHIK. 2000. Stability of macroinvertebrates to hydrological disturbance by flood and drought in a Brazilian semi-arid river (NE Brazil). Verh. Internat. Verein. Limnol., 27: 2461-2466.

SPONSELLER, R. A., E. F. BENFIELD \& H. M. VALETT. 2001. Relationships between land use, spatial scale and stream macroinvertebrate communities. Freshwater Biology, 46: 1409-1424.

STATISTICA (1984-85). Versión 5.0. StatSoft. Inc.

STATZNER, B., K. HOPPENHAUS, M. F. ARENS \& P. RICHOUX. 1997. Reproductive traits, habitat use and templet theory: a synthesis of worldwide data on aquatic insects. Freshwater Biology, 38: 109-135.

STOREY, A.W., D. H. D. EDWARD \& P. GAZEY. 1991. Surber and kick sampling: a comparison for the assessment of macroinvertebrate community 
structure in streams of south-western Australia. Hydrobiologia, 211: 111-121.

WILLIAMS, D. D. \& H. B. N. HYNES. 1976. The ecology of temporary streams. I. The faunas of two Canadian streams. Int. Revue ges. Hydrobiol., 61: 761-787.

WOOTTON, J. T., M. S. PARKER \& M. E. POWER. 1996. Effects of disturbance on river food webs. Science, 273:1558-1561.
ZAMORA-MUÑOZ, C., C. E. SÁINZ-CANTERO, A. SÁNCHEZ-ORTEGA \& J. ALBA-TERCEDOR. 1995. Are biological indices BMWP' and ASPT' and their significance regarding water quality seasonally dependent? factors explaining their variations. Water Res., 29: 285-290. 